\title{
Neurobrucellosis with Gait disturbance: A Neurological case report
}

\author{
Soraya Mehrabi ${ }^{1,2}$, Elahe shahriari ${ }^{1}$, Motahareh Afrakhteh ${ }^{3}$, \\ Mitra Ranjbar ${ }^{4}$, Marjan Zeinlai ${ }^{3}$, and Bahram Haghi \\ Ashtiani ${ }^{*}, 3$ \\ ${ }^{1}$ Department of Physiology, Faculty of Medicine, Iran \\ University of Medical Science, Tehran, Iran \\ ${ }^{2}$ Department of Advanced Technologies in Medicine, Iran \\ University of Medical Science, Tehran, Iran \\ ${ }^{3}$ Department of Neurology, Firouzgar Hospital, Iran University \\ of Medical Sciences, (IUMS), Tehran, Iran \\ ${ }^{4}$ Department of Infectious Disease, School of Medicine ,Iran \\ University of Medical Science \\ *Corresponding author: Bahram Haghi Ashtiani, \\ bahram.haghiashtiani@gmail.com
}

\section{August 16, 2020}

\begin{abstract}
Brucellosis is a multi-system infectious disease that exhibits with various manifestations and complications. Neurobrucellosis is a rare but serious presentation of brucellosis that can be discovered in every stages of the disease. Laboratory tests and physical examination and patient history are generally the basis for diagnosing the disease. It has both insidious and prolonged clinical course of the disease and long-term therapies. Also the most common pattern of exhibition is subacute or chronic. We reported a case of young female who had history of painless weakness in the right lower limb (proximal and distal) that started gradually and had progressed over the time, and after a month she felt weakness in the left lower limb with the same pattern. Lumbosacral Magnetic resonance imaging (MRI) with and
\end{abstract}


without contrast was shown evidence of enhancement thickening of caudal equina ventral roots. Brucella antigen titer was positive, the result was $1 / 160$. And other clinical tests were normal.

Patient treated with Intravenous injection (IV) Rifampicin and Intravenous Cotrimoxazole. patient was discharged with good health and continuinng all two medications for 5 months.

The descision was taken to report this case as a result of entire respond in patient's illness after a enduring disease. Neurobrucellosis is a treatable disease in which it would be better to consider a high indication of suspicion. Due to if ignored, it may cause signi cant morbidity and mortality.

Keywords:Neurobrucellosis, gait disturbance, accid paraprasia,weakness

\section{Introducton}

The most frequent bacterial zoonosis disease is Brucellosis and lead to more than 500,000 human infections per year worldwide.[1] Although, the disease has been reported all over the world, it has a higher widespread in countries where the health care problems and animal health are not standardized. In Asian countries and Turkey, a high prevalence of the disease has been reported.[2][3]

Di erent and non-speci c clinical manifestation make the diagnosis di cult. The most frequent symptoms are fever, myalgia, arthralgia, weight lossand night sweats.[2] Neurologic ndings of brucellosis occur in less than $5 \%$ in adolescents[4][5][6] but its incidence in pediatric is less than $0.8-1 \%$.[7][8]

Neurological complications include encephalitis, meningo encephalitis, radiculitis, myelitis, peripheral and cranial neuropathies, subarachnoid hemorrhage, psychiatric manifestations, brain abscess and demyelinating syndrome.[9] [10]

In endemic areas, if patient is discovered with neurological symptoms, diagnostic tests including: serum antibodies detection, isolation of cerebrospinal uid (CSF), and bone marrow must be performed. Examining the patient's complete history such as travel history, occupation and similar symptoms in other family members could also be very helpful to ruled out neurobrucellosis.[11] Analysis of CSF reveals an elevated protein concentration, moderate lukocy-tosis and hupoglycorrhachia.[11][12] CSF and blood cultures can be negative. Thus, the diagnosis is made by detecting brucella antibodies in CSF. This is 
diagnostic.[13]

We report an 13-year-old female patient with painless weakness in the both lower limb with same patterns, gait disturbance, accid paraprasia and loss of appetite. That has rarely been reported as a manifestation of neurobru-cellosis.

\section{Case Report}

A 13-year-old female patient was admitted in August in Firoozgar hospital, with history of gait distrurbance and loss of appetite without weight loss for 3 months. She'd had painless weakness in the right lower limb (proximal and distal) that started gradually and had progressed over the time, and after a month she felt weakness in the left lower limb with the same pattern. On arrival in the hospital, she had no fever and other parts of the vital signs were normal.No history of vomiting, headache, other sensorium, or seizure was reported. The patient belonged to a rural area and There is no history of contact with cows and goats and no raw milk has been consumed. Her vaccination program during infancy and childhood had been completely done and she had not any recent vaccination. Her parents were not relatives. Her father had treated brucellosis 20 years ago and her mother had been on treatment until 3 month ago. She had two younger sister and neither of them has the same symptoms.

On nervous system examination, our patient was conscious, Motor examination revealed normal muscle bulk, grade III power in proximal lower limb and grade II in distal and proximal force of lower limb. power of upper limb was normal. Deep Tendon Re ex (DTR) of upper limb was 2+, ankle and knee were 0 . Plantar response was donward bilatrally.Laboratory tests including Complete Blood Counts $(\mathrm{CBC})$, Blood Urea Nitrogen (BUN), serum creati-nine, electrolytes and liver function tests proved to be normal. Erythrocyte Sedimentation Rate (ESR) was 6 and C-Reactive Protein (CRP) 1. SSA-RO and SSB-LA both were 0.1 . Antibody for HIV virus, HBs Antigen and Anti HCV were negative. Lumbar puncture was performed and checked for anal-ysis (Table 1). Serological test results were positive(Table 2). Lumbosacral MRI with and without contrast was shown evidence of enhancement thicken-ing of caudal equina ventral roots (Fig.1).Because of normal Sensory Nerve Action Potential (SNAP) and decrease amplitude of Compound Muscle Ac-tion Potential(CMAP), the result of Electromyography (EMG) has shown subacute involvement of Anterior Horn Cell, especially in L5, S1 territory. 
Treatment was commence with Intravenous injection (IV) Rifampicin and In-travenous Cotrimoxazole. patient was discharged with good health and con-tinuinng all two medications (Rifampicin and Cotrimoxazole) for 5 months. She returned to hospital with recurrence symptoms due to discontinue med-ications before complete the course of treatment.

\begin{tabular}{cc}
\hline \hline \multicolumn{2}{c}{ Fluid Bodies } \\
\hline COMP $\stackrel{ }{\text { Test }}$ LETE CSF & Result \\
\cline { 2 - 2 } CSF & \\
W.B.C & 150 \\
R.B.C & 0 \\
Glucose & 19 \\
Total Protein & 152 \\
LDH & \\
LDH-P & 53 \\
Di Result & \\
Segment & 20 \\
Lymphocyte & 80 \\
\hline
\end{tabular}

Table 1: Pattern of CSF analysis

\begin{tabular}{cccc}
\hline Serology & Result & Unit & References \\
\hline Test & $1 / 160$ & Titer & Positive: $>=1 / 80$ \\
Wright & $1 / 160$ & Titer & Positive: $>=1 / 80$ \\
Coombs Wright & $1 / 80$ & Titer & Positive: $>=1 / 40$ \\
\hline ME_Wright(IgG)Titer & & & \\
Nodular reaction & Borderline & & \\
State & $7 \mathrm{~mm}$ & $\mathrm{~mm}$ & $\begin{array}{c}\text { Negetive: }<5 \mathrm{~mm} \\
\end{array}$ \\
& & & Borderline $: 5-10 \mathrm{~mm}$ \\
& & & Positive $:>10 \mathrm{~mm}$ \\
\hline
\end{tabular}

Table 2: Serological ndings 

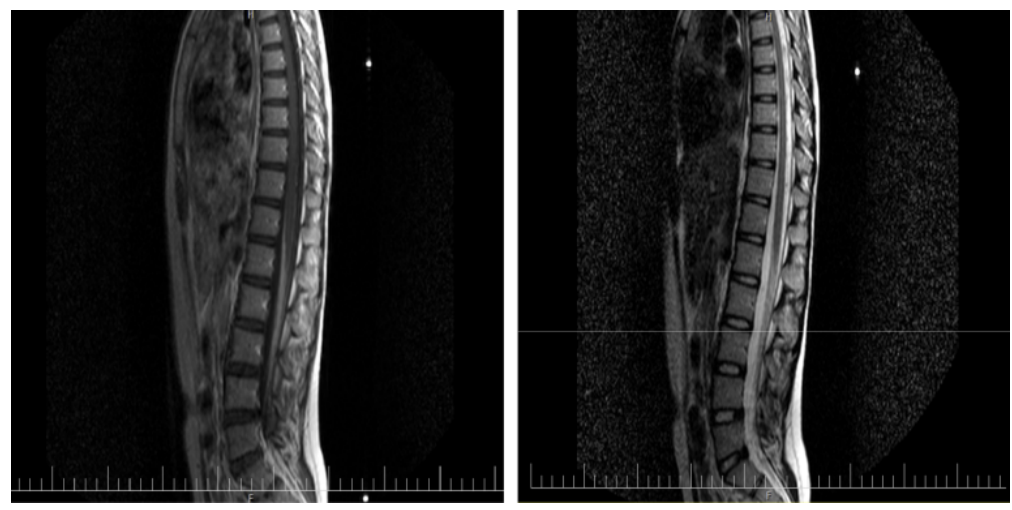

Figure 1: enhancement of caudal equina ventral roots

\section{Discussion}

It was obsereved that neurobrucellosis rarely reported on reviewing literature. Brucellosis is an endemic infectious disease in Iran. [14] Norobrucellosis accounts for about $3\{5 \%$ of cases of brucellosis.[11] The most common forms of neurobrucellosis are Meningitis and meningoencephalitis.[12] we reported a patient with gait disturbance, which is rare among reported cases.

The consumption of unpasteurized milk products is one of the most common cases of transmission of the disease.[15] yet our case, despite being rural, has no history of consumption of unpasteurized milk. Also Our patient presented with enhancement of caudal equina ventral roots in MR imaging and posi-tive serology test. In one study which carrid out in Saudi Arabia, they found clinical manifestation were related to the imaging abnormalities. They also categorized the changes of nervous system involvement of neurobrucellosis into 4 categories including: 1)normal, 2)in ammation (abnormal enhance-ment), 3) alteration of white matter,4) and radiologic ndigns which display vascular alteration.[16]

In another study,Gul HC, Erdem H, Gorenek L et al. from Turkey reported that the diagnosis was established by serum antibodies test and CSF ndings in 11 cases of neurobrucellosis.And they did not utilize imaging techniques 
to diagnose it.[17]

In treatment of this disease, due to the central nervous system is involved, antibiotics are used that are able to cross the blood-brain barrier well. It is preferable to use Co-trimoxazole, Doxycycline and Rifampicin. Combi-nation treatment with at least two medications of those indicated earlier is recommended.[2][12][18][18] The decision was taken to use Rifampicin and Co-trimoxazole for this patient.

In conclusion, Neurobrucellosis as an infectious disease, can be treated with a favorable result. The diagnosis of this disease is contingent generally upon high clinical attention in endemic areas such as Iran.The disease di ers in clinical diagnosis or radiology, especially in young patients with neurologi-cal disorders. Imaging neurobrucellosis results can be potentially misleading because they are di erent and can mimic other infectious, or in ammatory diseases like Tuberculosis. The sentence of what follows is drawn to the litr-erature Harrison's textbook of Medicine. Patients should ideally be followed clinically for up to 2 years because recurrence occurs in up to $30 \%$ of patients.

\section{Acknowledgements}

We would like to acknowledge the Neurology ward of Firoozgar Hospital for their kindly cooperation.

\section{References}

[1] Georgios Pappas, Photini Papadimitriou, Nikolaos Akritidis, Leonidas Christou, and Epameinondas V Tsianos. The new global map of human brucellosis. The Lancet infectious diseases, 6(2):91\{99, 2006.

[2] Hane Cem Gul, Hakan Erdem, and Semai Bek. Overview of neurobrucellosis: a pooled analysis of 187 cases. International Journal of Infectious Diseases, 13(6):e339\{e343, 2009.

[3] Osman Kizilkilic and Cem Calli. Neurobrucellosis. Neuroimaging Clinics, 21(4):927\{937, 2011. 
[4] EJ Young, GL Mandell, JE Bennett, and R Dolin. Brucella species principles and practice of infectious diseases. Churchil livingstone, 226, 2009.

[5] Donald R McLean, Neville Russell, and M Yousuf Khan. Neurobrucellosis: clinical and therapeutic features. Clinical infectious diseases, 15(4):582\{590, 1992.

[6] Gerald L Mandell, RG Douglas Jr, John E Bennett, et al. Principles and practice of infectious diseases. volumes 1 and 2. Principles and practice of infectious diseases. Volumes 1 and 2., 1979.

[7] YKR Habeeb, AKN Al-Najdi, SAH Sadek, and E Al-Onaizi. Paediatric neurobrucellosis: case report and literature review. Journal of infection, 37(1):59\{62, 1998.

[8] Aira Bucher, Peter Gaustad, and Eivind Pape. Chronic neurobrucellosis due to brucella melitensis. Scandinavian journal of infectious diseases, 22(2):223\{226, 1990.

[9] N Akritidis, M Bosilkovski, and G Pappas. Medical progress brucellosis. The New En. J. Med, 352:2325\{2336, 2005.

[10] Tumer Guven, Kenan Ugurlu, Onder Ergonul, Aysel Kocagul Celikbas, Sebnem Eren Gok, Selcuk Comoglu, Nurcan Baykam, and Basak Dokuzoguz. Neurobrucellosis: clinical and diagnostic features. Clinical Infectious Diseases, 56(10):1407\{1412, 2013.

[11] Raad A Shakir, ASN Al-Din, GF Araj, AR Lulu, AR Mousa, and MA Saadah. Clinical categories of neurobrucellosis: a report on 19 cases. Brain, 110(1):213\{223, 1987.

[12] Mahboubeh Haji-Abdolbagi, Mehrnaz Rasooli-Nejad, Sirous Jafari, Mehrdad Hasibi, and Abdolreza Soudbakhsh. Clinical and laboratory ndings in neurobrucellosis: review of 31 cases. Arch Iran Med, 11(1):21\{5, 2008.

[13] Daniel Tena, Alejandro Gonzalez-Praetorius, Alberto Lopez-Alonso, Jose Luis Pe na, Mar a Teresa Perez-Pomata, and Julia Bisquert. Acute meningitis due to brucella spp. European journal of pediatrics, 165(10):726, 2006.

[14] Saleh M Al Deeb, Basim A Yaqub, Hassan S Sharif, and Jayent G Phadke. Neurobrucellosis: clinical characteristics, diagnosis, and out-come. Neurology, 39(4):498\{498, 1989. 
[15] M Turgut, AT Turgut, and U Kosar. Spinal brucellosis: Turkish experience based on 452 cases published during the last century. Acta neurochirurgica, 148(10):1033\{1044, 2006.

[16] M Walid Al-Sous, Saeed Bohlega, M Zuheir Al-Kawi, Jehad Alwatban, and Donald R McLean. Neurobrucellosis: clinical and neuroimaging correlation. American Journal of Neuroradiology, 25(3):395\{401, 2004.

[17] Hane Cem Gul, Hakan Erdem, Levent Gorenek, Mehmet Fatih Ozdag, Yasin Kalpakci, Ismail Yasar Avci, Bulent Ahmet Besirbellioglu, and Can Polat Eyigun. Management of neurobrucellosis: an assessment of 11 cases. Internal Medicine, 47(11):995\{1001, 2008.

[18] J Bosch, J Linares, MJ Lopez de Goicoechea, J Ariza, MC Cisnal, and $R$ Martin. In-vitro activity of cipro oxacin, ceftriaxone and ve other antimicrobial agents against 95 strains of brucella melitensis. Journal of Antimicrobial Chemotherapy, 17(4):459\{461, 1986. 